\title{
Kochen-Specker theorem for a three-qubit system: a state-dependent proof with seventeen rays
}

\begin{abstract}
We consider Kochen-Specker theorem for three-qubit system with eight-dimensional state space. Reexamining the proof given by Kernaghan and Peres, we make some clarifications on the orthogonality of rays and rank-two projectors found by them. Basing on their five groups of orthogonal octad, we then show a proof that requires only seventeen rays.
\end{abstract}

Keyword: Kocehn-Specker theorem; Hidden variable; Qubit 